\title{
Chronic Mitral Regurgitation: When the Optimal Timing of Surgical Intervention?
}

\author{
H Oemar, K Yusoff, HB Abdulgani \\ Faculty of Medicine, Universiti Teknologi MARA, Selayang Hospital, 68100 Batu Caves, \\ Kuala Lumpur, MALAYSIA.
}

\begin{abstract}
Key words:

Optimal timing of surgery in mitral regurgitation $(M R)$ is a complex problem that has been studied Mitral widely. The pathophysiological mechanism and hemodynamic changes whereby MR exerts its regurgitation, Mitral valve surgery, Echocardiography. deleterious effects on survival is well recognized. Early reports in the literatures and newer prospective studies suggest that severe $M R$ is not a benign state and it has a high morbidity and eventually mortality. Thus, it is obviously rationale in understanding pathophysiological construct and be able to identify disease condition in choosing the golden moment for surgical intervention.

Surgical intervention has been exposed to be the only efficient management, but its optimal timing remains a matter of controversy. The ultimate goal of patient care is obviously no longer the relief of limiting symptoms but the achievement of an optimal long-term outcome with regard to mortality and morbidity. Preoperative developments of severe symptoms, left ventricular (LV) dysfunction, $L V$ enlargement, chronic atrial fibrillation, or progressive pulmonary hypertension were found to be associated with an unfavorable outcome.

The timing of surgical correction for MR depends chiefly on three factors: clinical symptoms, $L V$ function and the severity of MR. In term of waiting symptoms, the surgery has changed considerably from a relatively passive response to the development of severe symptoms, to an early surgery concept preceding the signs of $L V$ dysfunction. This because clinical symptoms can remain absent or minimal despite severe regurgitation caused by adaptive remodeling of $L V$ and left atrium, or because of patient adaptation of the disease. Thus, in chronic severe MR, there should be no waiting for LV function to decline before intervening, because the long-term results of that approach are not gratifying.

Recent data underscored that mitral surgery is associated with a considerably decreased subsequent risk of mortality and heart failure. The reduction in the risk of death associated with surgery is greater among patients with a larger effective regurgitant orifice (ERO) assessed echocardiographically than among those with a smaller ERO and results in normalization of the life expectancy. These data provide a firm basis for considering surgery in patients with asymptomatic chronic mitral regurgitation who have an ERO of at least $40 \mathrm{~mm}^{2}$.
\end{abstract}

(Cardiovasc. j. 2009; 1(2) : 142-147)

Mitral regurgitation (MR) is a common valvular heart disease characterized by abnormal reversal of blood flow from the left ventricle (LV) into the left atrium (LA). Chronic severe MR has both pathophysiologic and clinical consequences. The pathophysiologic results of MR are volume overload and resultant LV and LA remodeling and subsequent impairment in LV function. If the severe MR is uncorrected it would lead to excess mortality and morbidity. Even in asymptomatic patients with severe MR eventually need surgery during their follow up to evade indisposed consequences like LV dysfunction, arrhythmias, atrial fibrillation and excess mortality rate. The timing of surgical intervention depends on the consequences of existing severe MR as that compared with surgical outcomes.

The mitral valve apparatus is a complex anatomic and functional unit composed of the mitral annulus, valve leaflets, chorda tendineae, papillary muscles, and the underlying LV wall. Normal function depends on both normal anatomy of each of these components and on the overall three dimensional relationships of these structures to each other, including the effects of overall LV size, shape and systolic function. Diverse mechanisms of MR are associated with different clinical outcomes. In addition, medical or surgical treatment is directed at the specific mechanism of regurgitation in each 
individual patient. MR caused by an anatomic abnormality of the leaflets and chordae is termed primary regurgitation, while MR caused by a process primarily affecting the left ventricle is termed secondary MR. ${ }^{1}$

MR can be classified as mild, moderate and severe degree. Presently, in term of etiology, it divides into organic and functional MR. Organic MR means the abnormality is at the valve apparatus itself; however, in functional MR, the source of abnormality is at the poor function of myocardium and secondary to a dilated mitral annulus from dilatation of the left ventricle (LV). The ordinary causes of organic MR comprise mitral valve prolapse, rheumatic heart disease, coronary artery disease, infective endocarditis, certain drugs and collagen vascular disease. ${ }^{1,2}$

The ultimate goal of patient management is obviously no longer the relief of limiting symptoms but the achievement of an optimal long-term outcome with regard to mortality and morbidity. ${ }^{3}$ Yet, the management of MR and indications for surgery are still controversial. Previous outcome studies showed that patients with organic MR who have symptoms or a reduced ejection fraction are at high risk, warranting mitral surgery. Conversely, the clinical outcome among patients with asymptomatic MR is poorly defined, and criteria defining high-risk subgroups are uncertain. ${ }^{4}$ Such subgroups are important to identify as technical improvements in surgery allow the restoration of patients' life expectancy. ${ }^{5}$ Thus, surgery may be acceptable in high-risk patients with asymptomatic MR under medical management.

\section{Progressiveness of MR}

MR may worsen gradually over a prolonged period of time. Patients with mild to moderate MR may remain asymptomatic with little or no hemodynamic compromise for many years. However, MR from an organic MV abnormality tends to progress over time with an increase in volume overload due to an increase in regurgitant volume into the LA. ${ }^{1,2}$ Figure 1 demonstrates a moderate MR as assessed by Doppler echocardiography in a 50-year-old asymptomatic man. Calculated vena contracta of Color Doppler rose from $0.4 \mathrm{~cm}$ at the first visit become $0.8 \mathrm{~cm}$ at the following year of echocardiographic examination. However, his LV systolic function as well as end systolic diameter was not changed. This case subsequently was reffered to cardiac surgery for repair.

In patients with primary MR, there may be an interval of several years between the diagnosis of significant MR and onset of symptoms. In addition to development of symptoms, the two major concerns in patients with asymptomatic primary mitral valve disease are the risk of sudden death and the risk of irreversible left ventricular dysfunction. ${ }^{6}$
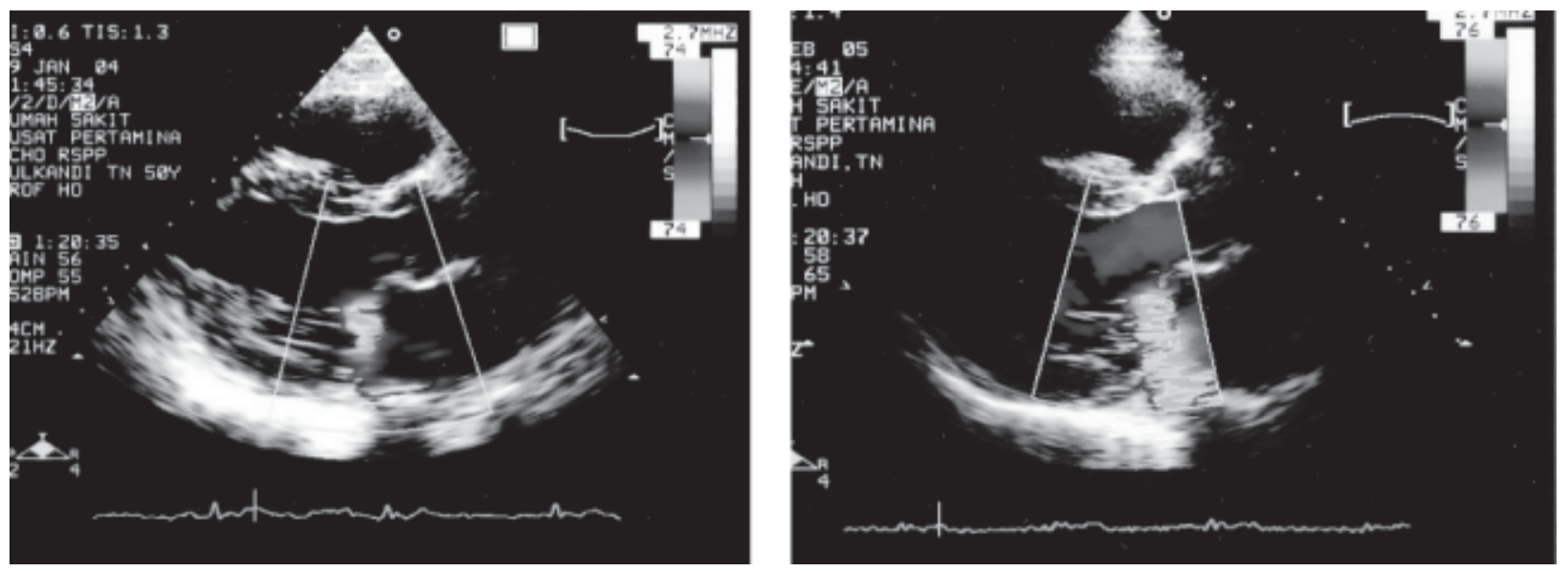

Fig.-1: Color Flow Doppler echocardiography of a 50-year-old man with asymptomatic MR presented with grade 2 severity with a VC of $0.4 \mathrm{~cm}$ (A). If became grade-3 after one year medical follow up with a VC of $0.8 \mathrm{~cm}(B) . V C=$ vena contractra. 
Chronic MR imposes a volume load on the left ventricle. In response to this volume load, initially the left ventricle empties more completely. Over time, left ventricular dilation maintains a normal forward cardiac output despite the regurgitant flow into the left atrium in systole. Early in the disease course, left ventricular systolic function remains normal, as measured by the rate of rise in ventricular pressure (dP/dt) or by end systolic maximum elastance (Emax). However, in the long term, some patients develop an irreversible decrease in left ventricular contractility. ${ }^{4}$

The progression of MR is essential in understanding this disease, because patients with severe MR incur a much higher mortality and morbidity than those with a milder degree of regurgitation. Furthermore, the high risk of LV dysfunction in patients with severe regurgitation has led to the suggestion that surgery should be performed early. ${ }^{2,4,5}$ However, because of the paucity of data available on progression of MR, the rates of change in the degree of $\mathrm{MR}$ and the underlying mechanisms are not certain. ${ }^{7}$

Major improvement of surgical techniques and surgical outcomes have led cardiologists and cardiac surgeons to become more aggressive in treating MR patients even they are asymptomatic or without LV dysfunction. Early editorial comment by Brian Griffin suggested that because MR is not a benign condition, if left without therapy, ultimately patient's morbidity and mortality increases ${ }^{8}$. On the other hand, however, contractile LV dysfunction may precede symptoms, and therefore evaluation of symptoms alone is inadequate in determining the time of surgery in chronic MR.

What information is required to decide a patient who would urge for surgery? There are factors that pose a big impact the outcome of patient with severe MR. Namely, symptoms, LV dysfunction and degree of MR.

\section{Wait for patient's symptoms?}

Once symptom occurs in patients with severe MR surgical intervention should be carried out. Because LV dysfunction has obviously preceded the symptoms. Patients with mild to moderate MR may remain asymptomatic and uncompromised for years, although a primary MV abnormality tends to progress over time. During this process, deleterious effects may be partially offset by beneficial compensatory changes.
For example, an increase in LV end-diastolic volume may lead to increased total stroke volume that helps restore cardiac output. Such compensation may permit a patient to remain asymptomatic for a longer period of time and even permit some patients to exercise briskly. However, prolonged severe volume overload eventually leads to LV dysfunction in most patients; typically, this occurs over the course of six to ten years in patient with chronic severe MR. ${ }^{9}$

Asymptomatic patients with mild MR and no evidence of LV enlargement, LV dysfunction, or pulmonary hypertension can be followed on an annual basis with instructions to alert the physician if symptoms develop in the interim. Yearly echocardiography is not necessary unless there is clinical evidence that MR has worsened.

In patients with moderate MR, clinical evaluation including echocardiography should be performed annually and sooner if symptoms occur. In asymptomatic patients with severe MR, clinical evaluation and echocardiography should be performed every six to 12 months to assess symptoms or transition to asymptomatic LV dysfunction. ${ }^{6,7,9}$

Waiting for severe symptoms is also creating problems, because symptoms always follows LV dysfunction. Severe symptoms associated with worse long-term survival and excess incidence of heart failure. The more severe pre-operative symptoms the lower post-operative EF and the higher incidence of heart failure during follow up (figure 2). When symptoms as a predictor, there is excess mortality in patients operated in with severe

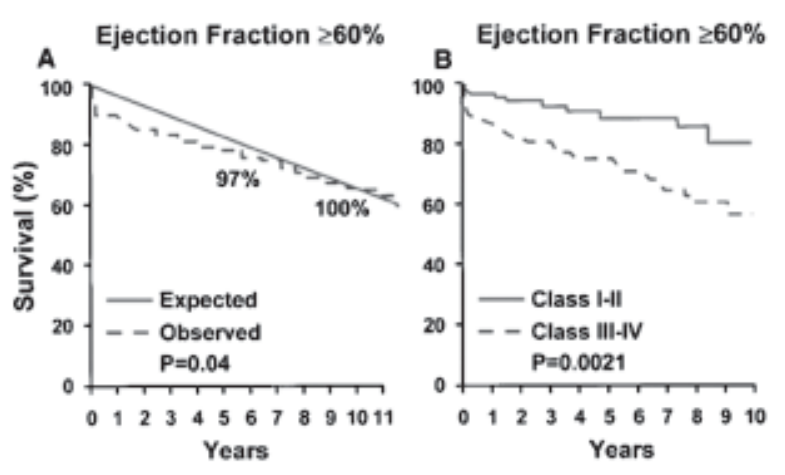

Fig.-2: Patients with asymptomatic and minimal symptoms (NYHA Class I-II) shows their survival rate was higher compared to those doing valve operation with significant symptomatic (NYHA IIIIV).(Adopted from Enrique-Sarano M.Ref. No.7) 
symptoms but no excess mortality in patients who had no or minimal symptoms, suggesting patients operated at an early stage with minimal symptoms (NYHA class I-II) have a better survival that patients with severe symptoms.

\section{Wait for LV dysfunction?}

Although interpretation of LV ejection fraction in patients with severe MR is difficult because the loading conditions facilitate ejection, several studies indicate that preoperative ejection fraction is an important predictor of postoperative survival in patients with chronic MR. ${ }^{7,9,10}$ Ejection fraction in a patient with MR with normal LV function is usually $\geq 60 \%$. Conversely, postoperative LV function is lower and survival reduced in patients with a preoperative ejection fraction $<60 \%$ compared with patients with higher ejection fractions. ${ }^{2}$ Because LV end-systolic dimension (or volume) may be less load dependent than ejection fraction, it can be used in timing of MV surgery. The guidelines suggest that end-systolic dimension (ESD) should be less than $40 \mathrm{~mm}$ preoperatively to ensure normal postoperative LV function. ${ }^{1,2,9}$

\section{Wait for MR became severe?}

Grading of mitral regurgitation is essential to justify the severity of MR in which surgical intervention may be offered to patients with severe MR even in the absence of overt LV dysfunction. Development of noninvasive quantitative Doppler echocardiography has transformed diagnostic approaches. Quantitative methods include assessment of regurgitant volume, regurgitant fraction and effective regurgitant orifice and the PISA method. MR is classified as severe when the regurgitant volume $>60 \mathrm{ml} /$ beat, Regurgitant fraction $>50 \%$ and ERO $>40 \mathrm{~mm}^{2}$ (Table I).

A study of Thomson and his colleagues suggested that the important proof is the presence of severe regurgitation as a potent indicator of timing of surgery. Because once severe regurgitation presented, the LV myocardial deterioration might obscurely occurred. ${ }^{6}$ Even patients without symptom or LVD, surgical intervention should be considered early in the course of MR. The similar results has been revealed by Enrique-Sarano and his colleagues with a prospective study comprised of 456 patients with asymptomatic organic MR. ${ }^{4}$
Table I.

Quantitative echo doppler for severity

\begin{tabular}{lccc}
\hline & Mild & Mod & Severe \\
\hline VC width $(\mathrm{cm})$ & $<0.3$ & $0.3-0.69$ & $\geq 0.7$ \\
R Vol $(\mathrm{ml} / \mathrm{beat})$ & $<30$ & $30-59$ & $\geq 60$ \\
$\mathrm{RF}(\%)$ & $<30$ & $30-49$ & $\geq 50$ \\
$\mathrm{ERO}\left(\mathrm{cm}^{2}\right)$ & $<0.20$ & $0.20-0.39$ & $\geq 0.40$ \\
\hline
\end{tabular}

Abbreviation: VC, vena contracta; $R$ Vol, regurgitant volume; $R F$, regurgitant fraction; $E R O$, effective regurgitant orifice. (Adopted from Zoghbi et al. JASE 2003;16:777)

Using Kaplan Meier statistical estimation of the survival among patients under medical management, this study showed that the highest 5 -years survival rate was among those with ERO of $<20 \mathrm{~mm}^{2}$, intermediate in those with an ERO between $20-39 \mathrm{~mm}^{2}$, and the lowest survival among those with an ERO $>40 \mathrm{~mm}^{2}$. Five-year rate of cardiac events were significantly different among the groups. Those with an ERO $<20 \mathrm{~mm}^{2}$ has a rate of $15 \%$, those with an ERO between 20-39 $\mathrm{mm}^{2}, 40 \%$; and those with an ERO of $>40 \mathrm{~mm}^{2}$ have highest cardiac event, $62 \%$. This study concludes that quantitative grading of $\mathrm{MR}$ is a powerful predictor of the clinical outcome of symptomatic MR. Patients with an effective regurgitant orifice of at least $40 \mathrm{~mm}^{2}$ should promptly be considered for cardiac surgery.

\section{Surgical Intervention}

The operative risks and improvements are important considerations in the appraisal of the timing of surgery. The operative mortality is of considerable importance but was too high to consider surgery in asymptomatic patients in the past. Thus, previously, surgical treatment of MR was avoided in patients with severe LV systolic dysfunction owing to concern about operative risk and peri-operative complications. ${ }^{1,2}$ However, for patients with organic MR, operative mortality was considerably decreased at present. ${ }^{9}$ More recently, with improvements in surgical techniques and increased knowledge of the benefit of cardioproctective regimen makes mortality rates to decline gradually.

Recent studies raised the possibility that the clinical course of initially asymptomatic organic MR may be more complicated than once 
thought. ${ }^{10,11}$ Additionally, clinical management is difficult and subject to controversy. Delaying surgical correction until symptoms appear is problematic, since the outcome may be suboptimal at that stage, with an excess risk of postoperative mortality and left ventricular dysfunction. Hence, it has been suggested that surgery for organic MR should be considered while patients are asymptomatic.

Enrique-Sarano and his colleagues reported that the reduction in the risk of death associated with surgery is greater among patients with a larger effective regurgitant orifice (EOA) than among those with a smaller effective orifice and results in normalization of the life expectancy. ${ }^{2,7}$ These data provide a firm basis for considering surgery in patients with asymptomatic MR who have an effective regurgitant orifice of at least $40 \mathrm{~mm}^{2}$. However, since the risk of atrial fibrillation increased after surgery, a clinical trial of surgery in such patients is essential. Therefore, quantitative grading of MR according to recent guidelines permits risk stratification of patients with isolated, asymptomatic, organic MR and should be part of the clinical decision-making process. $^{7}$

Current guidelines recommend surgery even if symptoms are still mild or when asymptomatic patients develop early signs of LV dysfunction, pulmonary hypertension, or atrial fibrillation. ${ }^{12}$ Within the background of ongoing controversy about the management of asymptomatic patients with severe degenerative MR. Rosenhek et al reported a study of watchful and wait of patients with asymptomatic till the criteria of symptom occur. ${ }^{3}$ Excellent outcome was achieved when patients were followed up carefully until either symptoms developed or until asymptomatic patients reached currently proposed criteria for surgery with regard to LV size, LV function, and pulmonary hypertension or until they developed recurrent atrial fibrillation.

The latest agreement among experts is patients who undergo surgery when already markedly symptomatic and those with reduced preoperative LV function were found to have particularly poor outcomes. ${ }^{1-3,12,13}$ It has been recognized that the prolonged burden of volume overload may have already resulted in irreversible myocardial damage and LV dysfunction when patients are still mildly symptomatic or even asymptomatic. Such observations suggest that surgery should be performed at an early stage. The best operative outcome has indeed been reported for patients who underwent surgery with no or only mild symptoms and an ejection fraction $\geq 0.60 .{ }^{13}$

\section{Clinical Implications and the Optimal Timing}

The timing of mitral surgery has changed considerably from a relatively passive response to the development of severe symptoms, to an early surgery concept preceding the signs of left ventricular dysfunction. The early surgery approach requires a high repair rate and a low operative mortality; therefore currently not all patients and not all institutions are candidates to apply the early indications of surgical correction of MR. Nevertheless, considerable progresses have recently been accomplished for the assessment and treatment of MR and surgery should be considered early in the course of the disease, when severe regurgitation has been diagnosed.

However, the price of maintaining normal left ventricular function and therefore survival in chronic asymptomatic MR is vigilance. ${ }^{8}$ The current guidelines for managing patients with severe chronic MR provide an adequate margin of safety to the patient when followed rigorously and conscientiously, including serial echocardiographic examinations, in experienced hands.

The guidelines offer a management strategy algorithm for patients with chronic severe MR. The algorithm outlines the clinical steps to take depending on the absence or presence of symptoms, the status of LV function, and more. ${ }^{10,12}$ For optimal survival, the guidelines emphasize that, unlike the timing of aortic valve replacement for aortic regurgitation, $L V$ ejection fraction should not be allowed to fall into the lower limit of normal in patients with chronic MR. However, clinicians often face the question as to whether the patient with advanced LV dysfunction is still a candidate for surgery. The guidelines recommend that surgery still be considered because even though a patient may experience persistent LV dysfunction, surgery will likely improve symptoms and prevent further LV Function deterioration. 


\section{References}

1. Otto CM. Timing of surgery in mitral regurgitation. Heart 2003; 89:100-5

2. Enriquez-Sarano M. Timing of mitral valve surgery. Heart 2002; 87:79-85

3. Rosenhek R, Rader F et.al. Outcome of watchful waiting in asymptomatic severe mitral regurgitation. Circulation 2006; 113:2238-44

4. Enriquez-Sarano M., Avierinos J., Messika-Zeitoun D et al. Quantitative Determinants of the Outcome of Asymptomatic Mitral Regurgitation. N. Eng. J Med. 2005; 352:875-83.

5. Tribouilloy CM, Enriquez-Sarano M, Schaff HV, Orszulak TA, Bailey KR, Tajik AJ et al. Impact of preoperative symptoms on survival after surgical correction of organic mitral regurgitation: rationale for optimizing surgical indications. Circulation 1999; 99:400-405.

6. Thomson HL, Enrique-Sarano M, Tajik AJ. Timing of surgery in patients with chronic, severe mitral regusrgitation. Cardiology in Review 2001; 9:137-43

7. Enriquez-Sarano M, Basmadjian A et al. Progression of Mitral Regurgitation A Prospective Doppler
Echocardiographic Study. J Am Coll Cardiol 1999; 34:1137- 44

8. Griffin BP. Timing of Surgical Intervention in Chronic Mitral Regurgitation: Is Vigilance enough? Circulation 2006; 113:2169-2172

9. Bonow RO. The $9^{\text {th }}$ Annual Cardiology at Cancun 2004 .

10. Cohn L, Couper G, Kinchla N et al. Decreased operative risk of surgical treatment of mitral regurgitation with or without coronary artery disease. J Am Coll Cardiol 1990; 16:1575-8.

11. Ling LH, Enriquez-Sarano M, Seward JB, Orszulak TA, Schaff HV, Bailey KR et al. Early surgery in patients with mitral regurgitation due to flail leaflets: a longterm outcome study. Circulation 1997; 96:1819 -1825.

12. Iung B, Gohlke-Barwolf C, Tornos P, Tribouilloy C, Hall R, Butchart $\mathrm{E}$ et al. Recommendations on the management of the asymptomatic patient with valvular heart disease. Eur Heart $J$ 2002; 23:1252-1266.

13. Ling LH, Enriquez-Sarano M, Seward JB, Orszulak TA, Schaff HV, Bailey KR et al. Early surgery in patients with mitral regurgitation due to flail leaflets: a longterm outcome study. Circulation 1997; 96:1819 -1825. 